\title{
Redox Buffering Capacity of Nanomaterials as an Index of ROS-based Therapeutics and Toxicity: A Preclinical Animal Study
}

Aniruddha Adhikari ${ }^{1}$, Susmita Mondal ${ }^{1}$, Monojit Das ${ }^{2,3}$, Ria Ghosh $^{4}$, Pritam Biswas ${ }^{5}$, Soumendra Darbar ${ }^{6}$, Soumendra Singh ${ }^{4}$, Anjan Kumar Das ${ }^{7}$, Siddhartha Sankar Bhattacharya ${ }^{2}$, Debasish $\mathrm{Pal}^{2}$, Asim Kumar Mallick ${ }^{8}$, Samir Kumar Pa1 ${ }^{1,2,4 *}$

\footnotetext{
${ }^{1}$ Department of Chemical, Biological and Macromolecular Sciences, S. N. Bose National Centre for Basic Sciences, Block JD, Sector 3, Salt Lake, Kolkata 700106, India

${ }^{2}$ Department of Zoology, Uluberia College, University of Calcutta, Uluberia, Howrah 711315, India

${ }^{3}$ Department of Zoology, Vidyasagar University, Vidyasagar University Road, Rangamati, Midnapore 721102, India

${ }^{4}$ Technical Research Centre, S. N. Bose National Centre for Basic Sciences, Block JD, Sector 3, Salt Lake, Kolkata 700106, India

${ }^{5}$ Department of Microbiology, St. Xavier's College, 30, Mother Teresa Sarani, Kolkata 700016, India

${ }^{6}$ Research and Development Division, Dey's Medical Stores (Mfg.) Pvt. Ltd., 62 Bondel Road, Ballygunge, Kolkata 700019, India

${ }^{7}$ Department of Pathology, Coochbehar Govt. Medical College and Hospital, Silver Jubilee Road, Coochbehar 736101, India

${ }^{8}$ Department of Pediatric Medicine, Nil Ratan Sirkar Medical College and Hospital, 138 AJC Bose Road, Sealdah, Rajabazar, Kolkata 700014, India
}

*Corresponding author's e-mail address: skpal@bose.res.in 


\begin{abstract}
Precise control of intracellular redox status, i.e., maintenance of physiological level of reactive oxygen species (ROS) for mediating normal cellular functions (oxidative eustress) while evading the excess ROS stress (distress) is central to the concept of redox medicine. In this regard, engineered nanoparticles with unique ROS generation, transition, or depletion functions have the potential to be the choice of redox therapeutics. However, it is always challenging to estimate whether ROS-induced intracellular events are beneficial or deleterious to the cell. Here, we propose the concept of redox buffering capacity as a therapeutic index of engineered nanomaterials. As a steady redox state is maintained for normal functioning cells, we hypothesize that the ability of a nanomaterial to preserve this homeostatic condition will dictate its therapeutic efficacy. Additionally, the redox buffering capacity is expected to provide information about the nanoparticle toxicity. Here, using citrate functionalized trimanganese tetroxide nanoparticles $\left(\mathrm{C}-\mathrm{Mn}_{3} \mathrm{O}_{4} \mathrm{NPs}\right)$ as a model nanosystem we explored its redox buffering capacity in erythrocytes. Furthermore, we went on to study the chronic toxic effect (if any) of this nanomaterial in animal model in order to co-relate with the experimentally estimated redox buffering capacity. This study could function as a framework for assessing the capability of a nanomaterial as redox medicine (whether maintains eustress or damages by creating distress), thus orienting its application and safety for clinical use.
\end{abstract}

\title{
KEYWORDS
}

Redox buffering; Redox medicine; Nanotherapy; Nanotoxicity; Redox homeostasis 


\section{INTRODUCTION}

Reactive oxygen species (ROS) and associated oxidative stress (so-called) remains the most fascinating paradigm for elucidating the adverse effects of engineered nanomaterials at cellular and molecular level [1]. ROS generating nanomaterials are generally considered cytotoxic for biomedical use, and their therapeutic applications are restricted to anticancer or anti-microbial activities [2-5]. Indeed, elevated ROS levels have the capacity to oxidize unsaturated fatty acids in lipids, and amino acids in proteins, inducing irreversible damage to vital organelles, and DNA, ultimately leading to cell apoptosis, and necrosis resulting in various chronic disorders including neurodegenerative, diabetes, and cardiovascular diseases [6-8]. However, recent understandings in free radical biology illustrate the pivotal role of ROS (at low concentration) as second messengers to modulate essential cellular functions like signaling, adhesion, migration, proliferation and homeostasis [9]. This inherent duality of ROS, the purposeful beneficial functions at physiological level (i.e., oxidative eustress), as well as, deleterious effects at supraphysiological concentration (i.e., oxidative distress) highlights the importance of maintaining proper redox balance for healthy organismal functions, and leads to the concept of redox medicine $[9,10]$. Redox medicine epitomize a promising tool for restoration of cell and tissue homeostasis via direct detoxification of reactive intermediates and/or by triggering cytoprotective and antioxidant signaling pathways [10]. In this sense, an interesting (perhaps the most important) avenue in redox medicine is the maintenance of oxidative eustress to regulate redox signaling and associated cell functions in addition to modulation of oxidative distress (i.e., inhibition to counteract pathological cell loss and induction to promote cell death of pathological cells). The disappointing history of efforts to prevent, or treat diseases with exogenous antioxidants (e.g., vitamin c, ascorbic acid, polyphenols etc.) further reflect the importance of redox-based therapeutics for clinical translation [11].

In this new front of redox medicine, ROS-based nanomedicines that involve nanomaterials with ROS-regulating properties, holds promise for optimized therapeutic efficacies [9]. Recent advancement in controlled synthesis process resulted into a wave of multifunctional nanomaterials with unique ROS generation, transition, or depletion functions. However, for in vivo application, it is a double-edged sword. After exerting expected therapeutic functions, 
the elevated ROS level may also impair adjacent normal tissues [5]. Moreover, if the nanomaterials are not degraded or excreted, their ROS-generating functions may still exist that may result in sustained oxidative damage. Therefore, balancing the therapeutic outcome and side effect of ROS generating nanomaterials is important for the optimization of therapeutic effects, which needs more careful characterization of the in vivo behavior of ROS and more exhaustive safety assessment of the nanomaterials [9]. In other words, information about ROS-mediated changes in cellular redox state, or ability of a nanomaterial to maintain eustress condition could be instrumental in dictating the therapeutic potential of a nanomaterial.

Unfortunately, the quantitative basis of changes in the intracellular redox state of the cells is not well-defined, thus leading to the dilemma that redox changes induced by oxidants in distinct cells types cannot be predicted [12]. The current state of the art measurement methods for estimating changes in cellular redox state is by measuring the concentration variation of intracellular redox couple GSSG/2GSH, and calculating the reduction potentials using Nernst equation, which is grossly misleading as spatial-temporal control exists between redox couples, and information about single couple cannot reflect the actual redox status [12-14]. Moreover, the method is cumbersome and prone to errors.

In this study, we introduce the concept of redox buffering capacity as a therapeutic index of engineered nanomaterials. As a steady redox state is maintained for normal functioning cells, we hypothesize that the ability of a nanomaterial to preserve this homeostatic condition will dictate its therapeutic efficacy. Additionally, the redox buffering capacity is expected to provide information about the nanoparticle toxicity. Here, using citrate functionalized trimanganese tetroxide nanoparticles $\left(\mathrm{C}-\mathrm{Mn}_{3} \mathrm{O}_{4} \mathrm{NPs}\right)$ as a model nanosystem we explored its redox buffering capacity in erythrocytes. In this regard it is worth mentioning that, $\mathrm{C}-\mathrm{Mn}_{3} \mathrm{O}_{4}$ NPs has previously been well studied from our group and showed therapeutic efficacy using both ROS generation and ROS scavenging abilities in in vitro as well as in vivo models. It treats hyperbilirubinemia by oxidative breakdown of bilirubin inside the body. On the other hand, it can mimic glutathione peroxidase to scavenge ROS and treat several diseases including hepatic fibrosis, heavy metal toxicity and Huntington's disorder. Thus, it is of considerable interest to assess the redox modulatory properties of this multifunctional nanoparticle. 
Furthermore, we went on to study the chronic toxic effect (if any) of this nanomaterial in animal model in order to co-relate with the experimentally estimated redox buffering capacity. This study could function as a framework for assessing the capability of a nanomaterial as redox medicine (whether maintains eustress or damages by creating distress), thus orienting its application and safety for clinical use. 


\section{RESULTS AND DISCUSSION}

\section{Redox Buffering Capacity of C-Mn $\mathrm{O}_{4}$ NPs.}

In the present study we used $\sim 10 \mathrm{~nm}$ nearly spherical C- $\mathrm{Mn}_{3} \mathrm{O}_{4} \mathrm{NPs}$ having monomodal size distribution (Figure 1). The optical properties of the nanoparticles have been thoroughly investigated and described by us in previous studies $[15,16]$. The nanoparticle has the unique ability to generate ROS in dark, without any photo- or chemi-activation [17]. Moreover, the NPs can function as a GSH-dependent GPx mimic to scavenge $\mathrm{H}_{2} \mathrm{O}_{2}$ in physiological milieu.

In order to explore the redox buffering effect of C- $\mathrm{Mn}_{3} \mathrm{O}_{4} \mathrm{NPs}$, we used the DCFH assay and measured the DCF intensity as a quantitative marker of ROS present in the reaction medium. First, we evaluated the in vitro redox buffering capacity of the synthesized NPs using $\mathrm{H}_{2} \mathrm{O}_{2}$ as an oxidant molecule. Figure $2 \mathrm{a}$ reflects the rate of $\mathrm{DCFH}$ oxidation with an increasing concentrations of $\mathrm{H}_{2} \mathrm{O}_{2}$ added to aqueous medium. The results clearly shows that the DCFH oxidation increased significantly with increasing concentration of $\mathrm{H}_{2} \mathrm{O}_{2}$. On the other hand, introduction of C- $\mathrm{Mn}_{3} \mathrm{O}_{4}$ NPs into the medium resulted into insignificant alteration in DCF intensity even after the addition of the highest concentration of $\mathrm{H}_{2} \mathrm{O}_{2}$. As shown in Figure 2a, the DCFH oxidation rate remained almost constant up to the addition of $2.5 \mathrm{mM}$ of $\mathrm{H}_{2} \mathrm{O}_{2}$. It signifies the efficient quenching of ROS generated from $\mathrm{H}_{2} \mathrm{O}_{2}$ by C- $\mathrm{Mn}_{3} \mathrm{O}_{4}$ NPs. It should be noted that $\mathrm{C}-\mathrm{Mn}_{3} \mathrm{O}_{4}$ NPs always maintained a constant ROS concentration in the reaction medium instead of eliminating all the ROS. Figure $2 \mathrm{a}$ depicts the relation between the DCFH oxidation rate $\left(\mathrm{V}_{\mathrm{f}}\right)$ and oxidant $\left(\mathrm{H}_{2} \mathrm{O}_{2}\right)$ concentration. Here, it is evident that DCFH oxidation by $\mathrm{H}_{2} \mathrm{O}_{2}$ follows an exponential kinetics and the oxidation rate clearly reduced in presence of $\mathrm{C}-\mathrm{Mn}_{3} \mathrm{O}_{4}$ NPs.

Next we quantified the in-vitro redox buffering capacity of the synthesized NPs. As per definition, the redox buffer capacity of a system is numerically equal to the magnitude of change of concentration of an oxidant (or a reductant) added to a solution, which is reduced or oxidized, to change the effective reduction potential by 1 unit $(1 \mathrm{~V})$ [12]. Thus, the redox buffer capacity $(\beta)$ can be represented as: 


$$
\beta=\frac{\delta \mathrm{c}_{\mathrm{ox}}}{\delta \mathrm{E}^{\mathrm{eff}}}
$$

Where $c_{o x}$ represents the concentration of the oxidant added to the system.

Experimentally, the redox buffering capacity and the effective reduction potential can be determined from the dependence of $1 / \mathrm{V}_{\mathrm{f}}$ on $1 / \mathrm{c}_{\mathrm{ox}}$ (Figure $2 \mathrm{~b}$ ). The correlation can be described by the following equation:

$$
\frac{1}{\Delta \mathrm{V}_{\mathrm{f}}}=\frac{1}{\Delta \mathrm{V}_{\mathrm{f}}^{\max }}+\frac{\mathrm{k}_{\mathrm{cap}}}{\Delta \mathrm{V}_{\mathrm{f}}^{\max }} \cdot \frac{1}{\mathrm{c}_{\mathrm{ox}}}
$$

Where, $\Delta \mathrm{V}_{\mathrm{f}}^{\max }$ is the highest rate of $\mathrm{DCFH}$ oxidation and $\mathrm{k}_{\text {cap }}$ is a constant having dimension of concentration. According to Eq. 2, the redox buffering capacity $\left(\frac{\mathrm{k}_{\mathrm{cap}}}{\Delta \mathrm{V}_{\mathrm{f}}^{\max }}\right)$ can be calculated from the tangent of slope angle of the plot of $1 / \mathrm{V}_{\mathrm{f}}\left(\mathrm{Y}\right.$-axis) vs. $1 / \mathrm{c}_{\mathrm{ox}}(\mathrm{X}$-axis).

Figure $2 \mathrm{~b}$ shows the significantly higher ( $\sim 1.3$ times) redox buffering capacity of $\mathrm{C}-\mathrm{Mn}_{3} \mathrm{O}_{4}$ NPs (1.29 unit) compared to Mili-Q water (0.97 unit).

To test the aforementioned redox buffering ability of $\mathrm{C}-\mathrm{Mn}_{3} \mathrm{O}_{4} \mathrm{NPs}$ in the cellular milieu we used human erythrocytes, or red blood corpuscle (RBC). The choice of erythrocytes was based on the fact that (i) their continuous exposure to high oxygen tensions, (ii) inability to replace damaged components, and (iii) the composition of membrane lipids partly of polyunsaturated fatty acid side chains that are vulnerable to peroxidation. Exogenously introduced $\mathrm{H}_{2} \mathrm{O}_{2}$ served as the inducer of oxidative stress. It is well-known that $\mathrm{H}_{2} \mathrm{O}_{2}$ generates massive oxidative stress and perturbs the intracellular redox state. For living cells, the electron donating and electron accepting capabilities of the intracellular medium can be specified by the value of effective redox potential, and any change in effective redox potential can be measured using specific redox sensor like DCFH in our study. Figure $2 \mathrm{c}$ and $2 \mathrm{~d}$ represent the in-cellulo redox buffering capacity of C- $\mathrm{Mn}_{3} \mathrm{O}_{4} \mathrm{NPs}$. It is evident from the results that the DCF intensity of the $\mathrm{RBCs}$ treated with C- $\mathrm{Mn}_{3} \mathrm{O}_{4} \mathrm{NPs}$ is significantly lower than the control $\mathrm{RBCs}$ treated with normal saline (Figure 2c). Following the method described above we found that 
the redox buffering capacity of C- $\mathrm{Mn}_{3} \mathrm{O}_{4}$ NPs (0.46 units) when integrated in erythrocytes is significantly higher ( 1.9 times) compared to the intrinsic cellular components ( 0.24 units) (Figure 2d). It has to be noted that the redox buffering capacity of the C- $\mathrm{Mn}_{3} \mathrm{O}_{4} \mathrm{NPs}$ in cellular system is considerably higher compared to in vitro aqueous system. This may be due to the additional (i.e., other than direct antioxidant activity) GPx mimicking activity of the $\mathrm{C}-\mathrm{Mn}_{3} \mathrm{O}_{4}$ NPs [18] which can only happen in presence of GSH, and intracellular metabolite.

Redox buffering requires sensing and rapid adjustment in the redox environment to maintain the oxidative eustress in cells. Cumulative data obtained from the experiments (both in-vitro and in-cellulo) suggest that $\mathrm{C}-\mathrm{Mn}_{3} \mathrm{O}_{4}$ NPs can sense and swiftly shift the redox state in the favour of oxidative eustress. This behaviour of $\mathrm{C}-\mathrm{Mn}_{3} \mathrm{O}_{4} \mathrm{NPs}$ as a redox modulator is consistent with that of $\mathrm{pH}$ buffers. $\mathrm{pH}$ buffers adjust the $\mathrm{pH}$ of the solution by sensing the concentration of $\mathrm{H}^{+}$ions present. Similarly, C- $\mathrm{Mn}_{3} \mathrm{O}_{4} \mathrm{NPs}$ perceive the amount of ROS in the medium and change its redox state accordingly. Also, it has been shown that a significant change in the concentration of C- $\mathrm{Mn}_{3} \mathrm{O}_{4} \mathrm{NPs}$ in the solution can cause a change in the redox state just like a $\mathrm{pH}$ buffer.

Previous studies suggest that for maintaining the oxidative eustress, C- $\mathrm{Mn}_{3} \mathrm{O}_{4} \mathrm{NPs}$ generate ROS from dissolved $\mathrm{O}_{2}$ and $\mathrm{H}_{2} \mathrm{O}$ through continuous disproportionation and comproportionation of the surface $\mathrm{Mn}^{2+} / \mathrm{Mn}^{3+} / \mathrm{Mn}^{4+}$ ions present at the spinel structure [15]. Electrons, produced from this process of conversion of $\mathrm{Mn}^{2+} \rightarrow \mathrm{Mn}^{3+} \rightarrow \mathrm{Mn}^{4+}$, react with the dissolved $\mathrm{O}_{2}$ and $\mathrm{H}_{2} \mathrm{O}$ of the medium and produce ROS. The ROS produced in this process is simultaneously neutralized by the conversion of $\mathrm{Mn}^{4+} \rightarrow \mathrm{Mn}^{3+} \rightarrow \mathrm{Mn}^{2+}$, i.e. the opposite pathway $[17,19,20]$. Therefore, when the cells stay at oxidative eustress condition, a dynamic equilibrium is maintained between the generation and elimination of ROS by C- $\mathrm{Mn}_{3} \mathrm{O}_{4} \mathrm{NPs}$. However, in many instances, the ROS level in the cells decreases resulting into immune suppression. During this condition the ROS generated by C- $\mathrm{Mn}_{3} \mathrm{O}_{4}$ NPs can take part in the cellular signalling process and maintain the normal cell functions. On the other hand, in oxidative distress conditions, the scenario becomes more complicated. The C- $\mathrm{Mn}_{3} \mathrm{O}_{4} \mathrm{NPs}$ can follow numerous buffering strategies to mitigate the oxidative distress. Besides quenching of ROS through the conversion of $\mathrm{Mn}^{2+} / \mathrm{Mn}^{3+} / \mathrm{Mn}^{4+}$, another likely strategy is that, in ROS excess medium, NPs act as a cofactor to some antioxidant enzymes and directly help 
intracellular anti-oxidant defence system [17, 20, 21]. Furthermore, the NPs can function as a GSH-dependent GPx mimic and scavenge excess ROS being incorporated into the cellular antioxidant enzyme network, as described in our previous study.

The combined results suggest that the $\mathrm{C}-\mathrm{Mn}_{3} \mathrm{O}_{4} \mathrm{NPs}$ can efficiently function as a redox buffer in intracellular condition. The described 'redox titration' method could be beneficial for comparing buffering capacity, as a marker of cyto-compatibility of different nanomaterials using minimum resources.

\section{$\mathrm{C}-\mathrm{Mn}_{3} \mathrm{O}_{4} \mathrm{NPs}$ show excellent hemocompatibility.}

Next we evaluated chronic toxicity of C- $\mathrm{Mn}_{3} \mathrm{O}_{4}$ NPs in animal model. With all newly developed nanoparticles, the potential for toxicity remains a factor in determining their specific applications in experimental small-animal, and ultimately clinical application. When NPs are administered in vivo, the most important physiological system they interact with is the blood and blood components. Then, if cleared by immune cells, they leave away from the bloodstream and reach the target organ [22]. Thus, it is important to measure the hemocompatibility of the NPs targeted for therapeutic use. We performed the hemolysis assay for evaluation of the hemolytic behaviors of C- $\mathrm{Mn}_{3} \mathrm{O}_{4}$ NPs on isolated mouse erythrocytes. The erythrocytes were first isolated by centrifugation and purified by sequential washes with sterile isotonic phosphate-buffered saline (PBS) $\mathrm{pH} 7.4$, then diluted to $5 \%$ hematocrit and incubated with different concentrations of NP suspensions $\left(0.01,0.05-\right.$ or $\left.0.1-\mathrm{ml} \mathrm{ml}^{-1} \mathrm{PBS}\right)$. The same amount of PBS was used as the negative control. An aqueous solution of RBCs was used as a positive control that is likely to show hemolysis. After 6 hrs incubation at room temperature, the samples were spun down to check the extent of hemolysis. Considering the hemolytic ability of positive control (water) as 100\%, all the NP treated samples showed negligible $(<5 \%)$ hemolysis (Figure $3 \mathrm{a}$ and $3 \mathrm{~b}$ ). Corresponding absorbance spectra (with the supernatant after final centrifugation) showed the optical features of C- $\mathrm{Mn}_{3} \mathrm{O}_{4} \mathrm{NPs}$ (Figure 3c) with ligand to metal charge transfer band at $300 \mathrm{~nm}$ and d-d transition bands at 429, 526 $\mathrm{nm}$. Only the positive control sample showed a distinct absorbance signature of hemoglobin with the Sorret band at $420 \mathrm{~nm}$ and Q-bands at 546 and $576 \mathrm{~nm}$ (Figure 3c). The scanning electron micrographs (SEM) (Figure 3e) of erythrocytes and Leishman stained whole blood smears supported the same (Figure 3f). The cell membrane remained unaltered after particle 
interaction, and the erythrocytes maintained normal biconcave shape just like control ones (Figure 3e \& 3f). To study the in vivo effect of C- $\mathrm{Mn}_{3} \mathrm{O}_{4} \mathrm{NPs}$ on blood, different hematology and serum biochemical indicators were measured after 90-days administration of the particles. All hematology marker values were within normal ranges and did not indicate a trend of toxicity associated with NPs (Supplementary Table S1). We further went on to assay ALAD activity, an important enzyme in the hematopoietic pathway which usually gets downregulated in cases of metal toxicity, particularly due to exposure of heavy metals like lead, mercury, etc. [23] However, we found no change in the activity of ALAD (Figure 3d). The hematoxylin and eosin stained spleen showed no abnormality (Figure 3g). These results indicate C- $\mathrm{Mn}_{3} \mathrm{O}_{4}$ NPs have excellent hemocompatibility.

\section{High dose of C-Mn $\mathrm{O}_{4}$ NPs cause mild oxidative damage to liver.}

The results of biodistribution (Supplementary Figure S1) study suggest the affinity of C$\mathrm{Mn}_{3} \mathrm{O}_{4}$ NPs towards the liver. The gross morphology (Figure 4a) and relative liver weights do not reveal any toxic effect on the organ. But compared to control animals, $0.5 \mathrm{mg} \mathrm{kg}^{-1} \mathrm{BW}$ NP-treated mice (not the other two groups of NP treated ones) displayed significantly higher serum alanine aminotransferase (ALT) and aspartate aminotransferase (AST) activity (Figure $4 \mathrm{~b} \& 4 \mathrm{c}, \mathrm{p}<0.001$ in one way ANOVA). A similar trend was observed in the case of alkaline phosphatase (ALP) and $\gamma$-glutamyl transferase ( $\gamma \mathrm{GT}$ ) activity (Figure $4 \mathrm{~d} \& 4 \mathrm{e}$ ), however, bilirubin levels showed no significant differences among groups (Figure 4f). The increased serum activity of transferases, normally confined within hepatic cells (not in serum), can be attributed to the damaged structural integrity of hepatocytes and subsequent release into the circulation. To know the real reason behind such elevated liver function parameters in the case of $0.5 \mathrm{mg} \mathrm{kg}^{-1} \mathrm{BW}$ NP-treated mice we went of histological analysis. In vehicle control animals, liver sections showed normal hepatic cells with well-preserved cytoplasm, prominent nucleus and nucleolus, and central vein (Figure $4 \mathrm{a}$ ). They along with 0.1 and $0.25 \mathrm{mg} \mathrm{kg}^{-1}$ BW NP-treated livers displayed characteristic hepatic architecture with hepatic plates directed from the portal triads toward the central vein where they freely anastomose. Inflammatory cells were seen intervening the hepatic strands and surrounding degenerative hepatocytes (Figure 4a). In addition, pericentral vein inflammation was also seen. Further histological damages including necrosis, ballooning degeneration, vacuolation, increased mitosis, and 
calcification were observed in the same group of mice (Figure $4 \mathrm{a}$ ). Thus, it is evident from the results of histopathological observations and liver function tests that $\mathrm{C}-\mathrm{Mn}_{3} \mathrm{O}_{4} \mathrm{NPs}$ at the highest administered dose $\left(0.5 \mathrm{mg} \mathrm{kg}^{-1} \mathrm{BW}\right)$ can cause moderate hepatic toxicity. Previously, we have demonstrated the NPs ability to generate reactive oxygen species (ROS) in aqueous solution. So, to understand the pathophysiological mechanism of observed toxicity, we measured plasma thiobarbituric acid reactive substances (TBARS) or malondialdehyde (MDA) content, a byproduct of lipid peroxidation and also an index of oxidative damage, which was found to be high in $0.5 \mathrm{mg} \mathrm{kg}^{-1} \mathrm{BW}$ NP-treated group (Figure 4i). We further extended our investigation to the changes in activities of superoxide dismutase (SOD), catalase (CAT) and glutathione peroxidase (GPX) which creates a mutually supportive team to fight against increased ROS [17, 24]. We found the downregulation of all the antioxidant enzyme activities in $0.5 \mathrm{mg} \mathrm{kg}^{-1} \mathrm{BW}$ NP-treated mice compared to control (Figure $4 \mathrm{j}-41$ ). The mitochondrial markers of oxidative damage like mPTP opening, membrane potential, and ATP content followed same trend (Figure 4m-4o). Though previous studies have shown that, $\mathrm{Mn}_{3} \mathrm{O}_{4}$ NPs can display antioxidant enzyme mimetic (particularly SOD and CAT) activity in the cellular environment, the humongous amount of ROS instantaneously generated due to high local concentration of NPs cannot be quenched through that mechanism. It should be noted that at the therapeutic dose $\left(0.25 \mathrm{mg} \mathrm{kg}^{-1} \mathrm{BW} \mathrm{NPs}\right)$ there is no evidence of oxidative damage.

\section{Moderate to high dose of C-Mn $\mathrm{O}_{4}$ NPs show mild to moderate inflammatory response in lungs.}

Sections of lung tissue from control and low dose C- $\mathrm{Mn}_{3} \mathrm{O}_{4} \mathrm{NP}$ treated animals have the normal appearance of fine lace because most of the lung is composed of thin-walled alveoli (Figure 5a). The alveoli are composed of a single layer of flattened epithelial cells and pneumocytes. Between the alveoli a thin layer of connective tissue and numerous capillaries are also lined with endothelium. However, the sections from moderate and high dose groups showed signs of nonspecific interstitial pneumonia in patchy manner other than normal lung architecture. The cellular pattern consists primarily of mild to moderate chronic interstitial inflammation, containing lymphocytes and a few plasma cells, in a uniform and patchy distribution. Signs of fibrosis, honeycombing, hyaline membranes and granulomas are absent. 
This kind of histological features may arise from the localization of particulate matters (as evident from our biodistribution study) in lungs [25]. This features are generally reversible and do not cause long term harm to the subjects [25].

\section{Effect of C-Mn ${ }_{3} \mathrm{O}_{4}$ NPs on kidney.}

Most of the drugs and xenobiotics pass through the kidney for excretion. But earlier studies have described that Mn cannot excrete through it. Our biodistribution results also revealed minimal deposition of Mn in the Kidney (Supplementary Figure S1). The histological analysis shows no damage and the normal structure of medulla and cortex was maintained in all four groups in spite of sexuality (Figure 5b). Consistent with these findings' other parameters like BUN, Creatinine, Urine Volume, Urine Protein and albumin to creatinine ratio remained similar to that of the control group (Supplementary Figure S2a-S2e).

\section{Effect of C-Mn $\mathrm{Mn}_{3} \mathrm{NPs}$ on reproductive organs.}

Affinity of nanoparticles towards testes is contradictory. Several nanoparticles such as gold and magnetic nanoparticles have been reported to enter testes in small quantities, whereas carbon nanotubes behave oppositely [26]. Our biodistribution results indicate that C- $\mathrm{Mn}_{3} \mathrm{O}_{4}$ NPs could enter or accumulate in the testis. The average testis weight (Supplementary Figure S3a), and morphology (Figure 5c) of NP-treated mice did not show any significant difference compared to the control mice. The observed accumulation of C- $\mathrm{Mn}_{3} \mathrm{O}_{4} \mathrm{NPs}$ in the testes upon chronic repeated dose administration have not shown any adverse effect on Sertoli cells, seminiferous tubules and germ cell nuclear volume (Supplementary Figure S3b-S3d). Figure $5 c$ presents hematoxylin and eosin stained cross-section structure of the testes of control and $\mathrm{C}-\mathrm{Mn}_{3} \mathrm{O}_{4} \mathrm{NP}$ treated mice. It is well known that within the seminiferous tubules, sperm cells are generated through differentiation of spermatozoa by meiosis. Microscopic images show normal testes structure for control mice (Figure 5c). Histologic examination of the cross sections of testes from all three dose group showed no alteration even after 90 days of administration (Figure 5c). Leydig cells or spermatids were similar in all groups. The sperm counts and sperm motility showed similar trends (Supplementary Figure S3e-S3g). We further microscopically observed eosin-Y stained sperms, and found slight deformity in $0.5 \mathrm{mg} \mathrm{kg}^{-1}$ BW NP treated group (Figure 5c-lower panel). Appropriate levels of male sex hormones are 
crucial for spermatogenesis. However, their functions can be affected by xenobiotics or foreign substances. Some endocrine toxicants, such as polychlorinated biphenyls and inorganic heavy metals, can alter the levels of these hormones, causing injury to testes, fault in spermatogenesis and male infertility. Notably nanoparticle-rich diesel exhaust inhalation disturbs the endocrine action of the male reproductive system by increasing the plasma testosterone levels in male rats. In this study, we determined the plasma concentrations of testosterone, LH and FSH on day 90 by enzyme-linked immunosorbent assay (ELISA). The results (Supplementary Figure S3h-S3j) indicate that the NPs did not alter plasma sex hormone levels in any of the groups.

Similar to male reproductive system, no observable sign of toxicity was found in case of female reproductive system. The haematoxylin and eosin stained sections of ovary from control animals displayed normal morphology (Figure 5d). Developing follicles (primordial, primary, pre-antral and antral follicles), corpora luteum, Graafian follicles and atretic follicles were found in the cortex of the ovary which was covered by a surface epithelium (Figure $5 \mathrm{~d}$ ). The ovary of $\mathrm{Mn}_{3} \mathrm{O}_{4}$ NPs treated mice (at any dose) did not show any noticeable changes in histology such as formation of fibrosis or cyst. The total number of healthy follicles (all categories) remains same after chronic exposure of $\mathrm{Mn}_{3} \mathrm{O}_{4}$ NPs across three dose groups (Supplementary Figure S4b). The mean number of corpora lutea in mice remained same in all groups. The estrus cycle was normal with typical distribution of diestrus, estrus, proestrus and metestrus stages (Supplementary Figure S3c \& S3d). The hormone levels showed no discrepancy (Supplementary Figure S3e-S3h).

\section{Effect of $\mathrm{C}-\mathrm{Mn}_{3} \mathrm{O}_{4}$ NPs on glucose metabolism and pancreas}

We further investigated the effect of $\mathrm{C}-\mathrm{Mn}_{3} \mathrm{O}_{4}$ NPs on plasma glucose levels and its metabolism. Microscopic investigation of the pancreas, the major organ responsible for glucose homeostasis, both control, and NP-treated mice showed normal pancreatic tissue forming characteristic pancreatic acini with basal nuclei and amphiphilic cytoplasm (Supplementary Figure S5a). The islet of Langerhans's displayed islet cells arranged in trabecular and acinar pattern with abundant eosinophilic cytoplasm and central small nucleus, separated by thin loose connective tissue with thin vessels. Islets have their typical spherical shape with a large number of $\beta$-cells with normal round shape distinct round nuclei. 
No significant change was observed across the groups for total cholesterol, triglyceride, LDL, HDL or VLDL (Supplementary Figure S5c-S5f). Plasma glucose levels (12h fasting), $\mathrm{HbA}_{1 \mathrm{c}}$ and serum insulin concentration remained similar to control animals (Supplementary Figure $\mathrm{S} 5 \mathrm{~g} \& \mathrm{~S} 5 \mathrm{e}) .2 \mathrm{~h}$ glucose tolerance test showed indistinctive results among the groups with identical AUC (Supplementary Figure S5i \& S5j). Thus, the NPs neither affects glucose metabolism nor changes the lipid profile in the experimental regime. 


\section{CONCLUSION}

In summary, the present study intends to highlight the efficient redox buffering capability of an exclusively developed C- $\mathrm{Mn}_{3} \mathrm{O}_{4}$ NPs. The chronic toxicity study implies its potential safety. Furthermore, our study can serve as a template to assess other redox modulatory nanotherapeutic agents. The method is easy, semi-quantitative and sustainable for robust use. To the best of our understanding this may open a new avenue in preliminary screening and evaluation of toxicity in the redox based nanomedicine research.

\section{ACKNOWLEDGEMENT}

The authors thank SAIF-IIT Bombay for ICP-AES measurements. MD thanks University Grants Commission (UGC), Govt. of India for Junior Research Fellowship. SKP thanks the Indian National Academy of Engineering (INAE) for the Abdul Kalam Technology Innovation National Fellowship, INAE/121/AKF. The authors thank the Department of Biotechnology (DBT, West Bengal) for the financial grant under BOOST scheme, 339/WBBDC/1P-2/2013.

\section{CONFLICT OF INTEREST}

The authors disclose no conflict of interest.

\section{DATA AVAILABILITY}

All data that support the findings of this study are available within the published article (and supplementary information files). Study related additional data will be available from SKP upon legitimate request. 


\section{MATERIALS AND METHODS}

\section{Quantification \& characterization of in vitro ROS.}

DCFH a very popular chemical for quantification of ROS generation was prepared from DCFH-DA via de-esterification reaction at room temperature following a standardized protocol described in earlier studies [17]. ROS generated in the reaction medium reacted with DCFH and convert it to DCF which has a characteristic fluorescence emission maxima at $520 \mathrm{~nm}$ when excited at $488 \mathrm{~nm}$. DCFH was allowed to react with increasing concentration of Ch- $\mathrm{Mn}_{3} \mathrm{O}_{4}$ NPs in aqueous medium and fluorescence was recorded using fluorolog, Model LFI-3751 (Horiba-Jobin Yvon, Edison, NJ) spectrofluorimeter.

\section{Measurement of intracellular ROS generation.}

After isolation RBC were incubated with C- $\mathrm{Mn}_{3} \mathrm{O}_{4} \mathrm{NPs}\left(3.4 \mu \mathrm{g} \mathrm{ml}^{-1}\right)$ for 3 hours. After the incubation cells were washed with PBS and treated with DCFH-DA probe for 30 minutes. At the end of this period the excess amount of DCFH-DA was thoroughly rinsed with PBS. Then cells were scraped and suspended in PBS. The cell suspensions was subjected to the measurement of DCF fluorescence intensity using fluorolog, Model LFI-3751 (Horiba-Jobin Yvon, Edison, NJ) spectrofluorimeter.

All spectroscopic experiments were performed using quartz cuvette of path length $1 \mathrm{~cm}$.

\section{Animals.}

Swiss albino mice of both sex (2-3 weeks, weighing 12-15 g) were used for the current study. Four or five mice were housed together in polypropylene cage under a 12:12 light-dark cycle. During the time, food and water were accessible ad libitum. Mice were procured from National Institute of Nutrition, Hyderabad, India. All animal studies and experimental procedures were performed at animal house of Uluberia College, West Bengal, India (Reg. No.2057/GO/ReRcBi/S/19/CPCSEA) following the protocol approved by the Institutional Animal Ethics Committee (Ethical Clearance No. - 02/S/UC-IAEC/01/2019) as per standard guideline of CPCSEA, New Delhi, India. 


\section{Biochemical tests.}

At the end of the experimental period, the animals were euthanized and decapitated after being fasted. Blood was collected from retro orbital plexus just before sacrifice, kept in sterile non-heparinized tubes in slanting position for $45 \mathrm{~min}$ and centrifuged at $3500 \times \mathrm{g}$ for $20 \mathrm{~min}$. The clear serum was obtained and used in subsequent biochemical analysis.

Biochemical evaluations were performed using commercially available kits (Autospan Liquid Gold, Span Diagnostic Ltd., India) following the protocol described by respective manufacturers. $\delta$-aminolevulinic acid dehydratase ( $\delta$-ALAD) activity was measured using the European standardized method [27]. Complete blood count (CBC) was obtained using an automated cell counter (Medonic CA 620, Boule Diagnostics, Sweden). Hormones were estimated using commercially available ELISA kits following the protocol described by respective manufacturers.

Tissue samples were collected, homogenized in cold phosphate buffer (0.1 M; pH 7.4), and centrifuged at 10,000 r.p.m. at $4^{\circ} \mathrm{C}$ for $15 \mathrm{~min}$. The supernatants were collected and used to determine the activity of SOD, CAT, GPx and GSH as well as the content of malondialdehyde (MDA) as marker of lipid peroxidation. Lipid peroxidation was determined in TBARS formation using a reported procedure [28, 29]. SOD (Sigma, MO, USA), CAT (Abcam, Germany), and GPx activities (Sigma, MO, USA) were estimated using commercially available test kits following protocols recommended by respective manufacturers.

\section{Histology.}

For microscopic evaluation, a conventional technique of paraffin wax sectioning and differential staining was used [30]. Organs were removed following incision, fixed in 10\% neutral buffered formalin saline for $72 \mathrm{~h}$, dehydrated in graduated ethanol (50-100\%), cleared in xylene, and embedded in paraffin. Microtome was used to prepare ultrathin sections (4-5 $\mu \mathrm{m})$, followed by staining with hematoxylin and eosin (H\&E). Histopathological changes were examined under the microscope (Olympus BX51) equipped with a CCD based camera. 


\section{Biodistribution.}

ICP-AES (ARCOS, SPECTRO Analytical Instruments GmbH, Germany) was carried out to determine the amount of $\mathrm{Mn}$ in blood and other organs like liver, kidney, spleen and, brain. The open acid digestion method was used for sample preparation. In brief, tissues were dried using liquid nitrogen and weighted. The freeze-dried samples were dissolved in an acid mixture that contained $\mathrm{HNO}_{3}(3 \mathrm{ml}), \mathrm{H}_{2} \mathrm{SO}_{4}(2 \mathrm{ml})$, and $\mathrm{H}_{2} \mathrm{O}_{2}(1 \mathrm{ml})$, heated at $120^{\circ} \mathrm{C}$ until only a residue remained, and then diluted with deionized water to $10 \mathrm{ml}$.

\section{Statistical analysis.}

All quantitative data are expressed as Mean \pm SD unless otherwise stated. One-way analysis of variance (ANOVA) followed by Tukey's multiple comparison test was executed for comparison of different parameters between the groups using a computer program GraphPad Prism (version 8.00 for Windows), GraphPad Software (CA, USA). p<0.05 was considered significant. 


\section{REFERENCES}

1. Stone V, Donaldson K: Signs of stress. Nature Nanotechnology 2006, 1(1):23-24.

2. Wang S, Li F, Hu X, Lv M, Fan C, Ling D: Tuning the Intrinsic Nanotoxicity in Advanced Therapeutics. Advanced Therapeutics 2018, 1(5):1800059.

3. Xia T, Kovochich M, Brant J, Hotze M, Sempf J, Oberley T, Sioutas C, Yeh JI, Wiesner MR, Nel AE: Comparison of the Abilities of Ambient and Manufactured Nanoparticles To Induce Cellular Toxicity According to an Oxidative Stress Paradigm. Nano Letters 2006, 6(8):1794-1807.

4. Nel A, Xia T, Mädler L, Li N: Toxic Potential of Materials at the Nanolevel. Science 2006, 311(5761):622-627.

5. Yang B, Chen Y, Shi J: Reactive Oxygen Species (ROS)-Based Nanomedicine. Chemical Reviews 2019, 119(8):4881-4985.

6. Winterbourn CC: Reconciling the chemistry and biology of reactive oxygen species. Nature Chemical Biology 2008, 4(5):278-286.

7. Wang Z, Zhang Y, Ju E, Liu Z, Cao F, Chen Z, Ren J, Qu X: Biomimetic nanoflowers by self-assembly of nanozymes to induce intracellular oxidative damage against hypoxic tumors. Nature Communications 2018, 9(1):3334.

8. Trachootham D, Alexandre J, Huang P: Targeting cancer cells by ROS-mediated mechanisms: a radical therapeutic approach? Nature Reviews Drug Discovery 2009, 8(7):579-591.

9. Sies H, Jones DP: Reactive oxygen species (ROS) as pleiotropic physiological signalling agents. Nature Reviews Molecular Cell Biology 2020, 21(7):363-383.

10. Sims CM, Hanna SK, Heller DA, Horoszko CP, Johnson ME, Montoro Bustos AR, Reipa V, Riley KR, Nelson BC: Redox-active nanomaterials for nanomedicine applications. Nanoscale 2017, 9(40):15226-15251.

11. Nathan C, Cunningham-Bussel A: Beyond oxidative stress: an immunologist's guide to reactive oxygen species. Nature Reviews Immunology 2013, 13(5):349-361.

12. Martinovich GG, Martinovich IV, Cherenkevich SN, Sauer H: Redox Buffer Capacity of the Cell: Theoretical and Experimental Approach. Cell Biochemistry and Biophysics 2010, 58(2):75-83.

13. Morgan B, Ezerina D, Amoako TNE, Riemer J, Seedorf M, Dick TP: Multiple glutathione disulfide removal pathways mediate cytosolic redox homeostasis. Nature Chemical Biology 2013, 9(2):119-125.

14. Martinovich GG, Cherenkevich SN, Sauer H: Intracellular redox state: towards quantitative description. European Biophysics Journal 2005, 34(7):937-942. 
15. Giri A, Goswami N, Sasmal C, Polley N, Majumdar D, Sarkar S, Bandyopadhyay SN, Singha A, Pal SK: Unprecedented catalytic activity of Mn3O4 nanoparticles: potential lead of a sustainable therapeutic agent for hyperbilirubinemia. $R S C$ Advances 2014, 4(10):5075-5079.

16. Adhikari A, Biswas P, Mondal S, Das M, Darbar S, Hameed AM, Alharbi A, Ahmed SA, Sankar Bhattacharya S, Pal D et al: A Smart Nanotherapeutic Agent for in vitro and in vivo Reversal of Heavy-Metal-Induced Causality: Key Information from Optical Spectroscopy. ChemMedChem 2020, 15(5):420-429.

17. Adhikari A, Polley N, Darbar S, Bagchi D, Pal SK: Citrate functionalized Mn3O4 in nanotherapy of hepatic fibrosis by oral administration. Future Science $O A$ 2016, 2(4):FSO146.

18. Adhikari A, Mondal S, Das M, Biswas P, Pal U, Darbar S, Bhattacharya SS, Pal D, Saha-Dasgupta T, Das AK et al: Incorporation of a Biocompatible Nanozyme in Cellular Antioxidant Enzyme Cascade Reverses Huntington's Like Disorder in Preclinical Model. Advanced Healthcare Materials 2020:2001736.

19. Horsburgh MJ, Wharton SJ, Karavolos M, Foster SJ: Manganese: elemental defence for a life with oxygen. Trends in Microbiology 2002, 10(11):496-501.

20. Takashima T, Hashimoto K, Nakamura R: Inhibition of Charge Disproportionation of MnO2 Electrocatalysts for Efficient Water Oxidation under Neutral Conditions. Journal of the American Chemical Society 2012, 134(44):18153-18156.

21. Adhikari A, Das M, Mondal S, Darbar S, Das AK, Bhattacharya SS, Pal D, Pal SK: Manganese neurotoxicity: nano-oxide compensates for ion-damage in mammals. Biomaterials Science 2019, 7(11):4491-4502.

22. Huang X, Li L, Liu T, Hao N, Liu H, Chen D, Tang F: The Shape Effect of Mesoporous Silica Nanoparticles on Biodistribution, Clearance, and Biocompatibility in Vivo. ACS Nano 2011, 5(7):5390-5399.

23. Adhikari A, Darbar S, Chatterjee T, Das M, Polley N, Bhattacharyya M, Bhattacharya S, Pal D, Pal SK: Spectroscopic Studies on Dual Role of Natural Flavonoids in Detoxification of Lead Poisoning: Bench-to-Bedside Preclinical Trial. ACS Omega 2018, 3(11):15975-15987.

24. Adhikari A, Polley N, Darbar S, Pal SK: Therapeutic Potential of Surface Functionalized Mn3O4 Nanoparticles Against Chronic Liver Diseases in Murine Model. Materials Focus 2017, 6(3):280-289.

25. Kumar V, Abbas AK, Fausto N, Aster JC: Robbins and Cotran pathologic basis of disease, professional edition e-book: Elsevier health sciences; 2014.

26. Bai Y, Zhang Y, Zhang J, Mu Q, Zhang W, Butch ER, Snyder SE, Yan B: Repeated administrations of carbon nanotubes in male mice cause reversible testis damage without affecting fertility. Nature Nanotechnology 2010, 5(9):683-689. 
27. Fujita H: Measurement of $\boldsymbol{\delta}$-Aminolevulinate Dehydratase Activity. Current Protocols in Toxicology 1999, 1(1):8.6.1-8.6.11.

28. Moore K, Roberts LJ: Measurement of Lipid Peroxidation. Free Radic Res 1998, 28(6):659-671.

29. Garcia YJ, Rodríguez-Malaver AJ, Peñaloza N: Lipid Peroxidation Measurement by Thiobarbituric Acid Assay in Rat Cerebellar Slices. J Neurosci Methods 2005, 144(1):127-135.

30. Polley N, Saha S, Adhikari A, Banerjee S, Darbar S, Das S, Pal SK: Safe and Symptomatic Medicinal Use of Surface-Functionalized Mn3O4 Nanoparticles for Hyperbilirubinemia Treatment in Mice. Nanomedicine 2015, 10(15):2349-2363. 


\section{Figures and Tables}
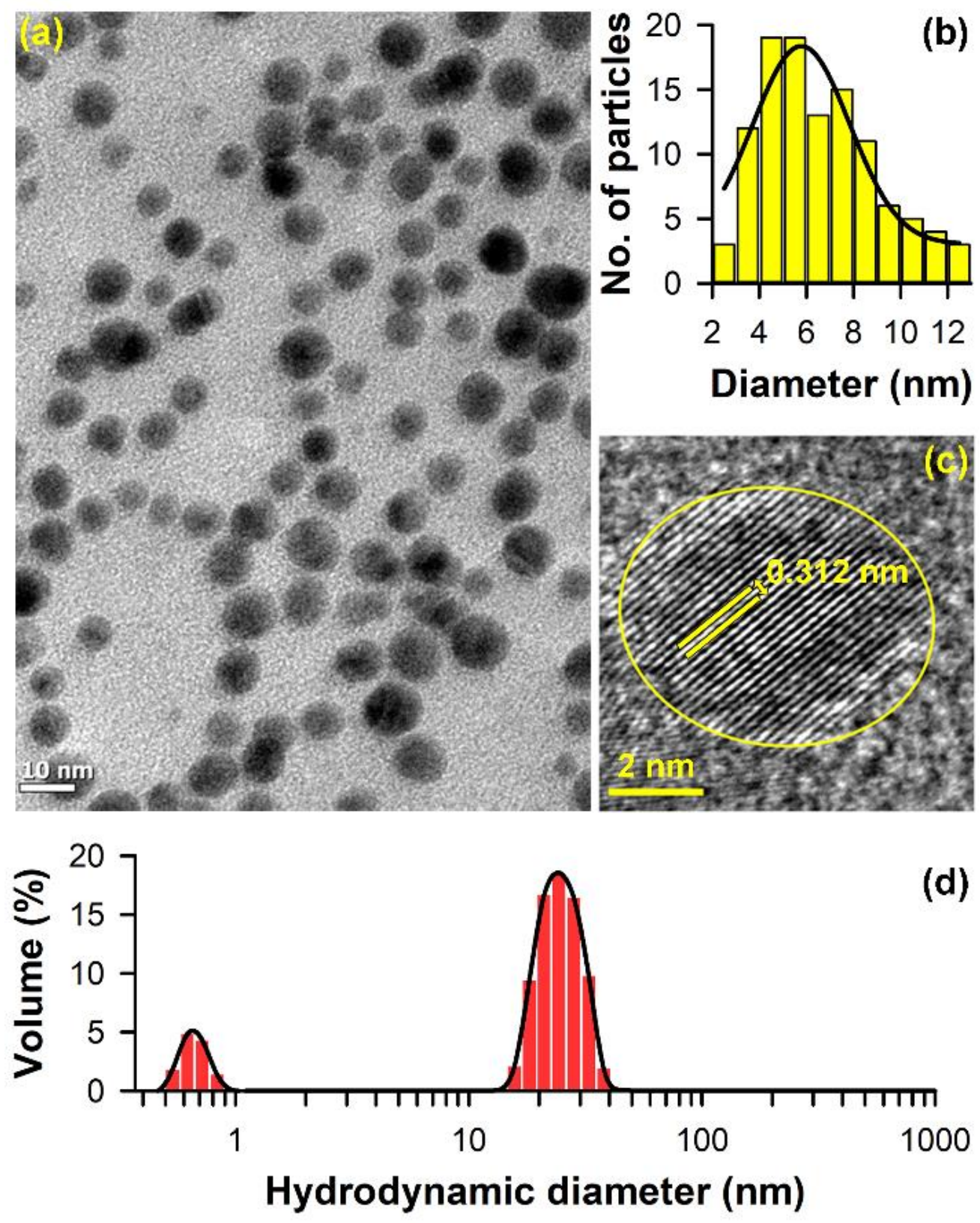

Figure 1. Properties of citrate- $\mathrm{Mn}_{3} \mathrm{O}_{4}$ nanoparticles $\left(\mathrm{C}-\mathrm{Mn}_{3} \mathrm{O}_{4} \mathrm{NPs}\right)$. (a) Transmission electron microscopic (TEM) images of C- $\mathrm{Mn}_{3} \mathrm{O}_{4}$ NPs. (b) The particle size distribution of the NPs with average size of $6.25 \pm 2.41 \mathrm{~nm}$. (c) High resolution TEM (HR-TEM) of single NP shows the interplanar distance of $0.312 \mathrm{~nm}$ for 112 planes of $\mathrm{C}-\mathrm{Mn}_{3} \mathrm{O}_{4}$ NPs. (d) Hydrodynamic diameter of $\mathrm{C}-\mathrm{Mn}_{3} \mathrm{O}_{4} \mathrm{NPs}$ as measured by DLS. 

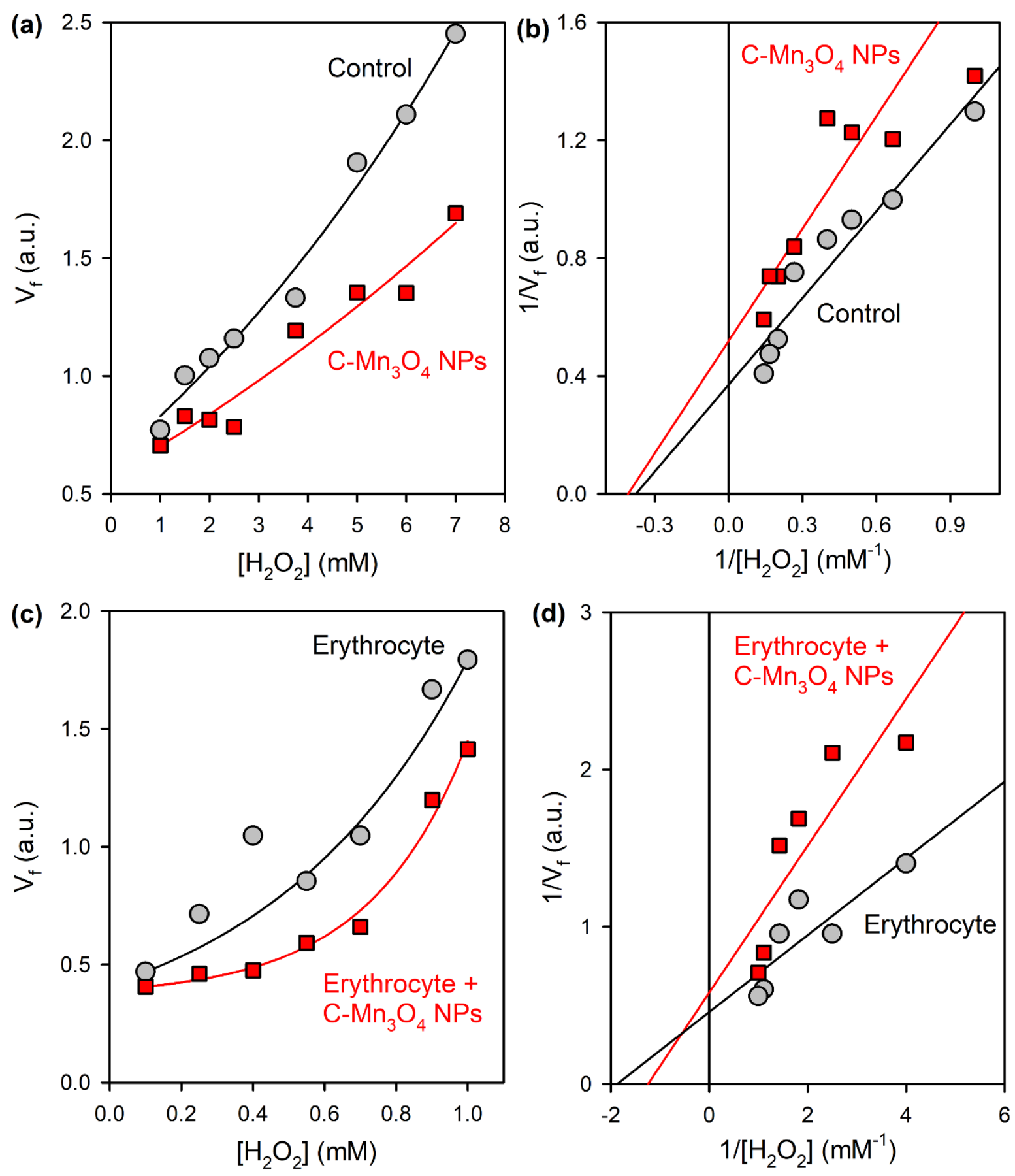

Figure 2. Redox buffering capacity of C-Mn $\mathrm{O}_{4} \mathrm{NPs}$. (a) Rate of DCFH oxidation in presence of $\mathrm{H}_{2} \mathrm{O}_{2}$. (b) Double reciprocal plot of the same. (c) Rate of $\mathrm{DCFH}$ oxidation in presence of $\mathrm{H}_{2} \mathrm{O}_{2}$ in erythrocytes. (d) Double reciprocal plot of the same. 

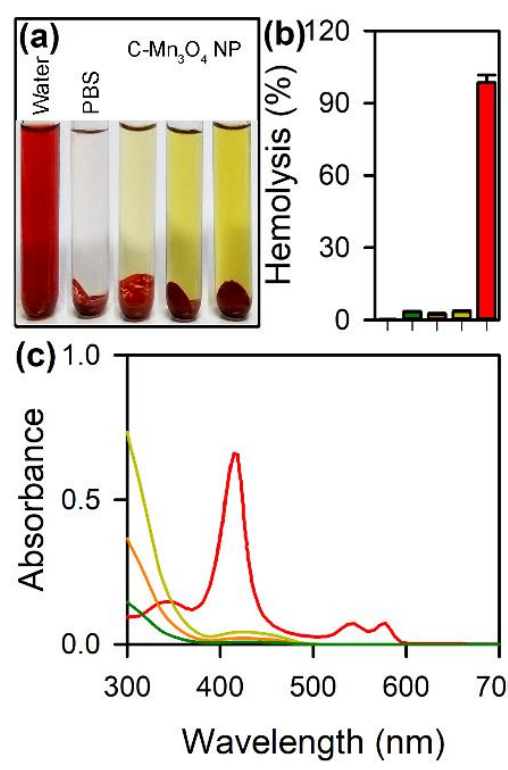

(e)

Control \begin{tabular}{cc}
$\mathrm{C}-\mathrm{Mn}_{3} \mathrm{O}_{4} \mathrm{NP}\left(\mathrm{mg} \mathrm{kg}^{-1} \mathrm{BW}\right)$ \\
\hline 0.25 & 0.50
\end{tabular}
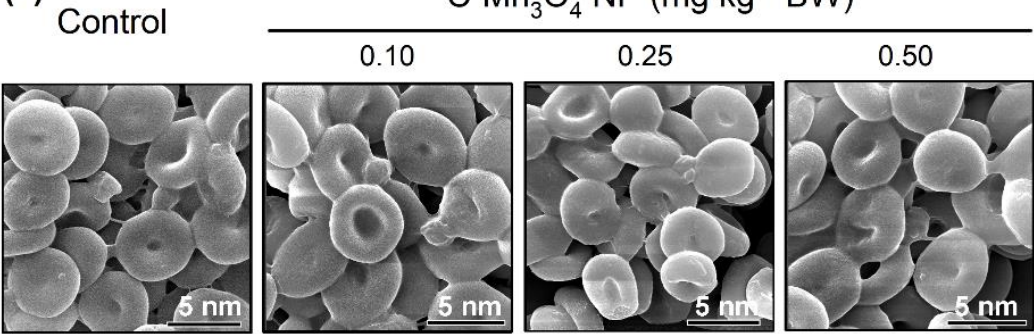

(f) Whole Blood

(Leishman stain)
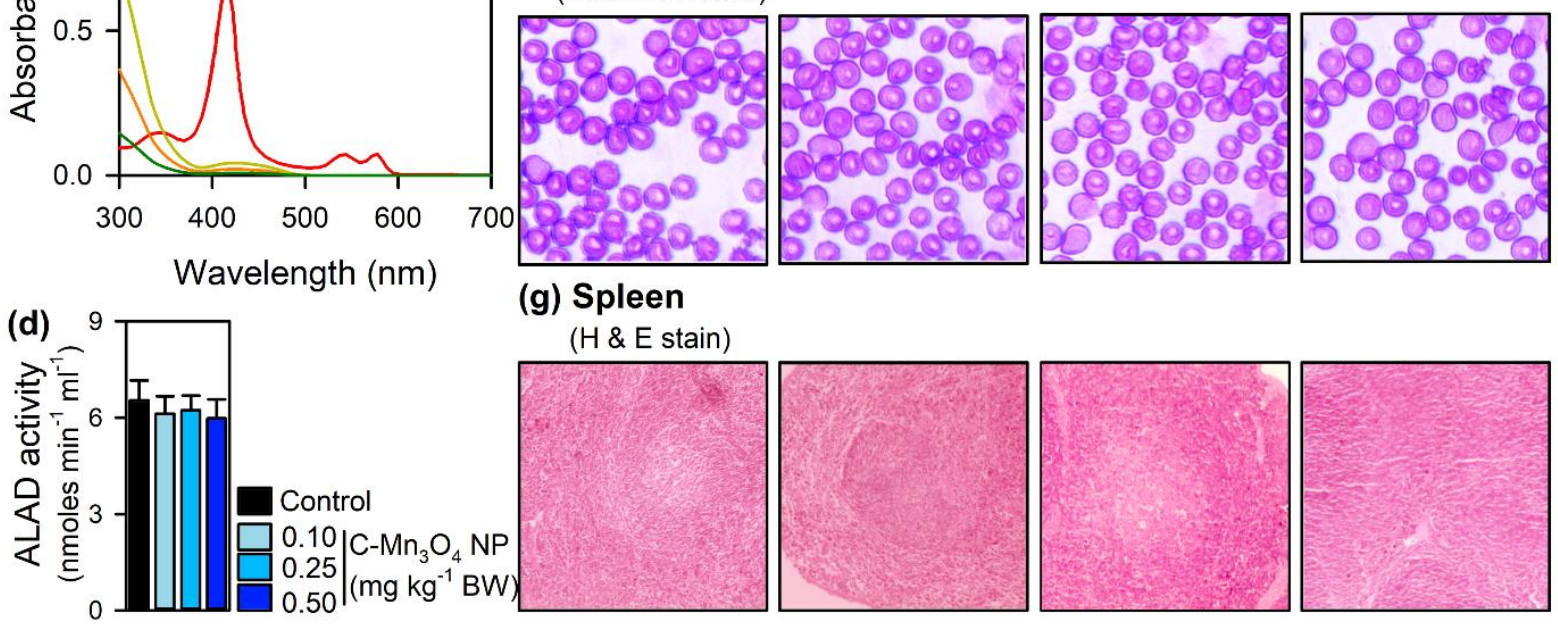

(g) Spleen

(H \& E stain)
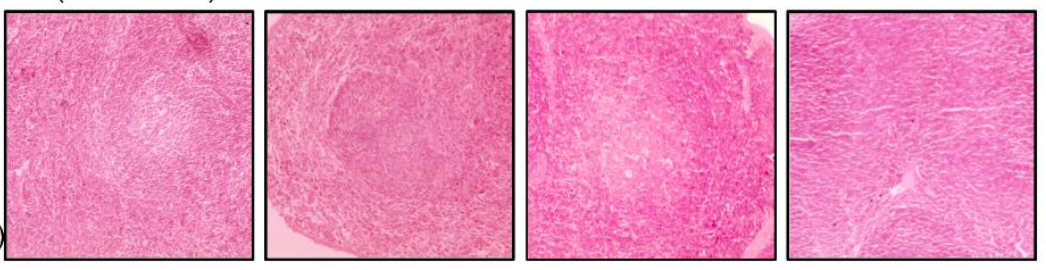

Figure 3. Effect of C-Mn ${ }_{3} \mathrm{O}_{4}$ NPs on haematological system. (a) Visual inspection of the tubes containing RBC after exposure to NPs. No visible haemolytic behaviour was observed for NPs, water was used as positive control and PBs as negative control. The bar plot shows percentage of haemolysis induced by C- $\mathrm{Mn}_{3} \mathrm{O}_{4}$ NPs. (c) UV-vis absorbance spectra of the supernatant. (d) ALAD activity. (e) Scanning electron micrographs of the $\mathrm{RBCs}$ incubated with $\mathrm{C}-\mathrm{Mn}_{3} \mathrm{O}_{4}$ NPs shows no visible damage to the membrane or morphology. (f) Leishman stained whole blood from the BALB/c mice treated with different doses of NPs for 90 days. (g) H\&E stained spleen sections after 90 days' oral administration of NPs.

All data represented as Mean \pm Standard Deviation (SD). $\mathrm{N}=6$ for each measurement. 

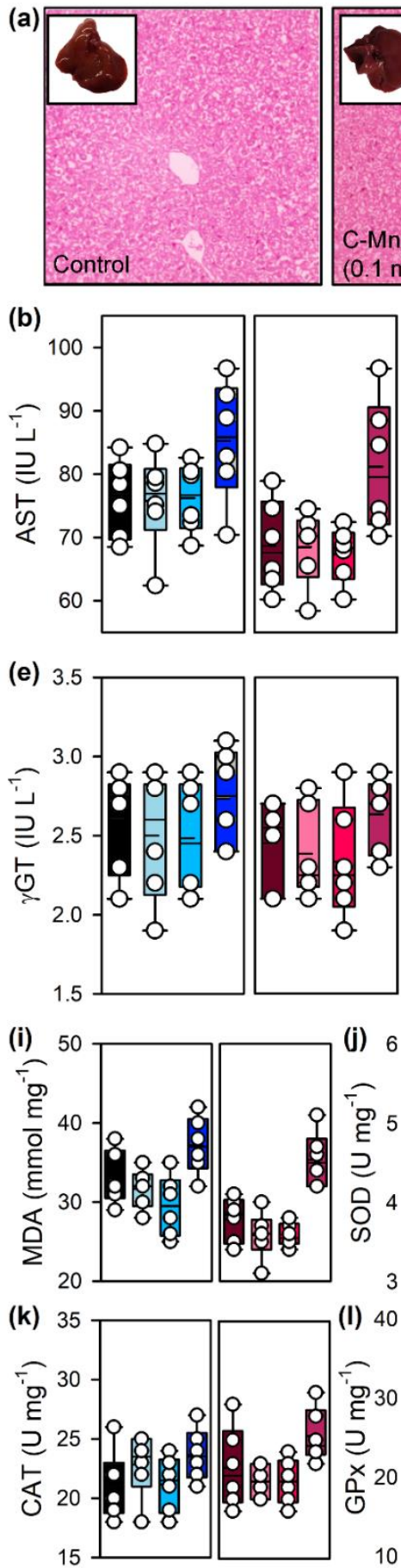
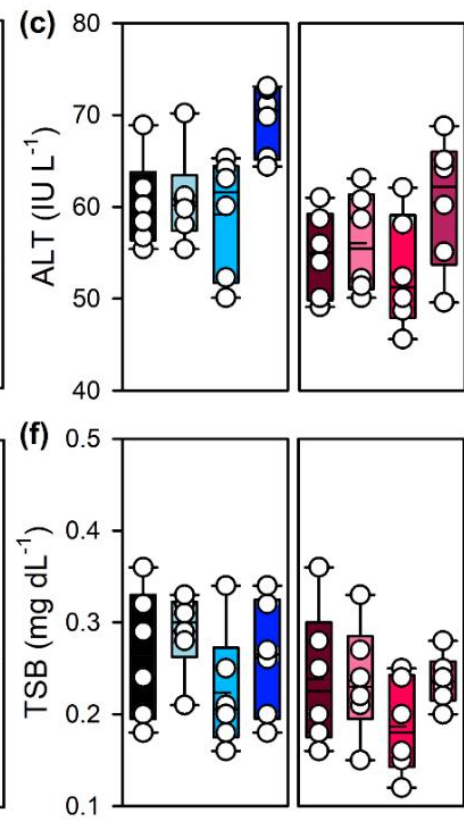

(m)

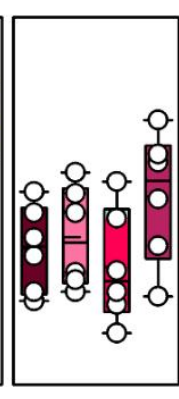

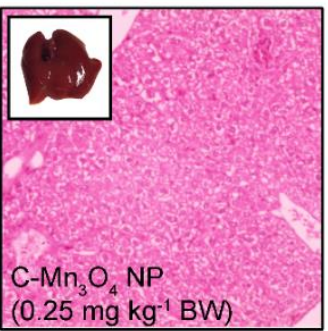
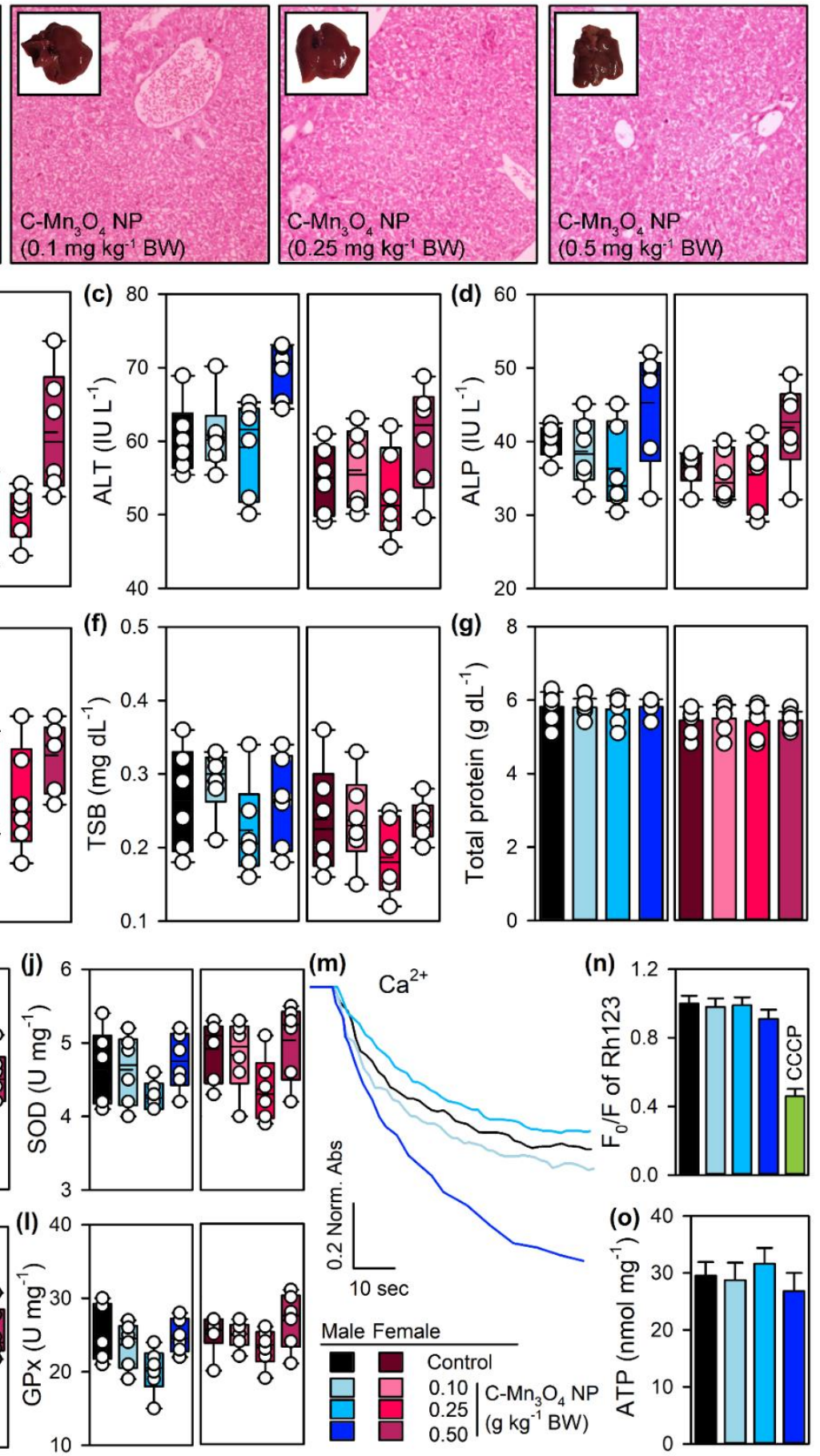

Figure 4. Effect of C-Mn $\mathrm{Mn}_{3} \mathrm{O}_{4} \mathrm{NPs}$ on hepatic function after chronic administration. (a) Morphology of the liver and H\&E stained liver sections. (b-g) Effect on liver function parameters show chronic administration of high dose of NPs can cause damage hepatic cells. However, the therapeutic dose has no detrimental effect. (i-1) Assessment of cellular antioxidant enzymes revealed the damage to be ROS mediated. (m-o) Effect of the NPs on mitochondria reveals membrane depolarization along with increased MPTP which in turn increase cellular apoptosis through release of cytochrome c.

All data represented as Mean \pm Standard Deviation (SD). $\mathrm{N}=6$ for each measurement. 
(a) Lungs

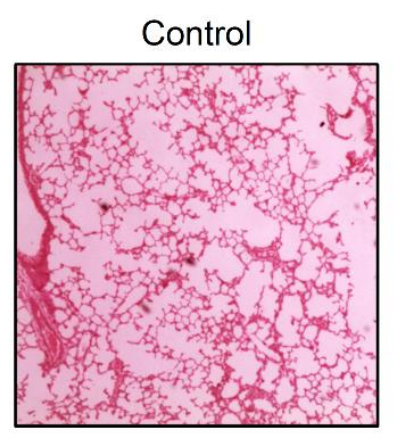

\section{(b) Kidney}

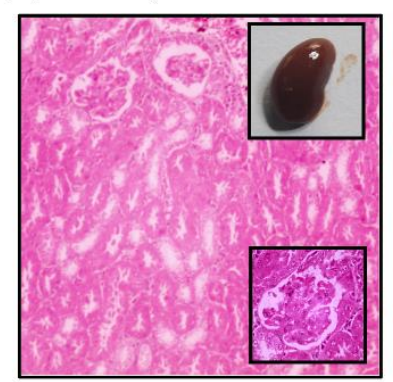

(c) Testes
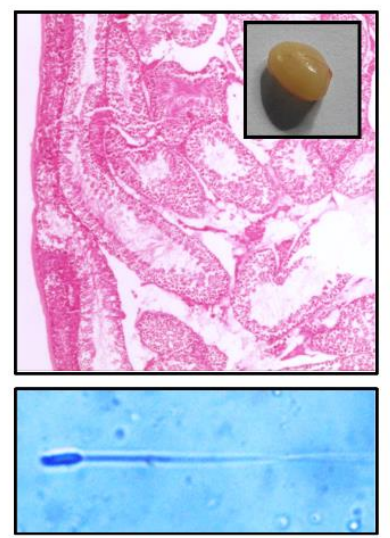

(d) Ovary

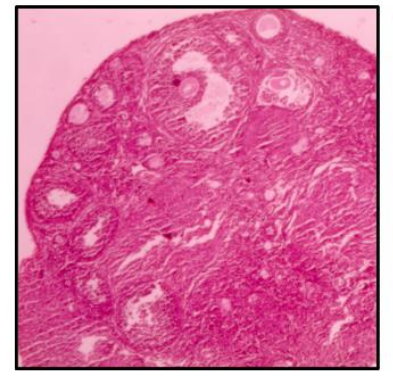

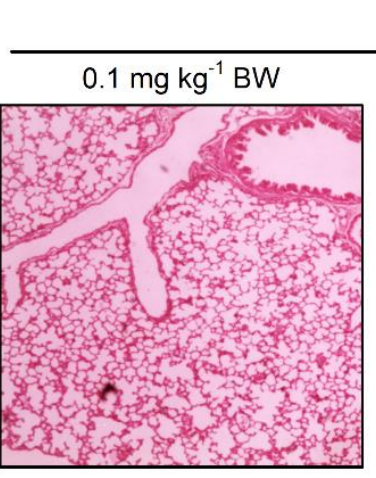

$\mathrm{C}-\mathrm{Mn}_{3} \mathrm{O}_{4} \mathrm{NP}$
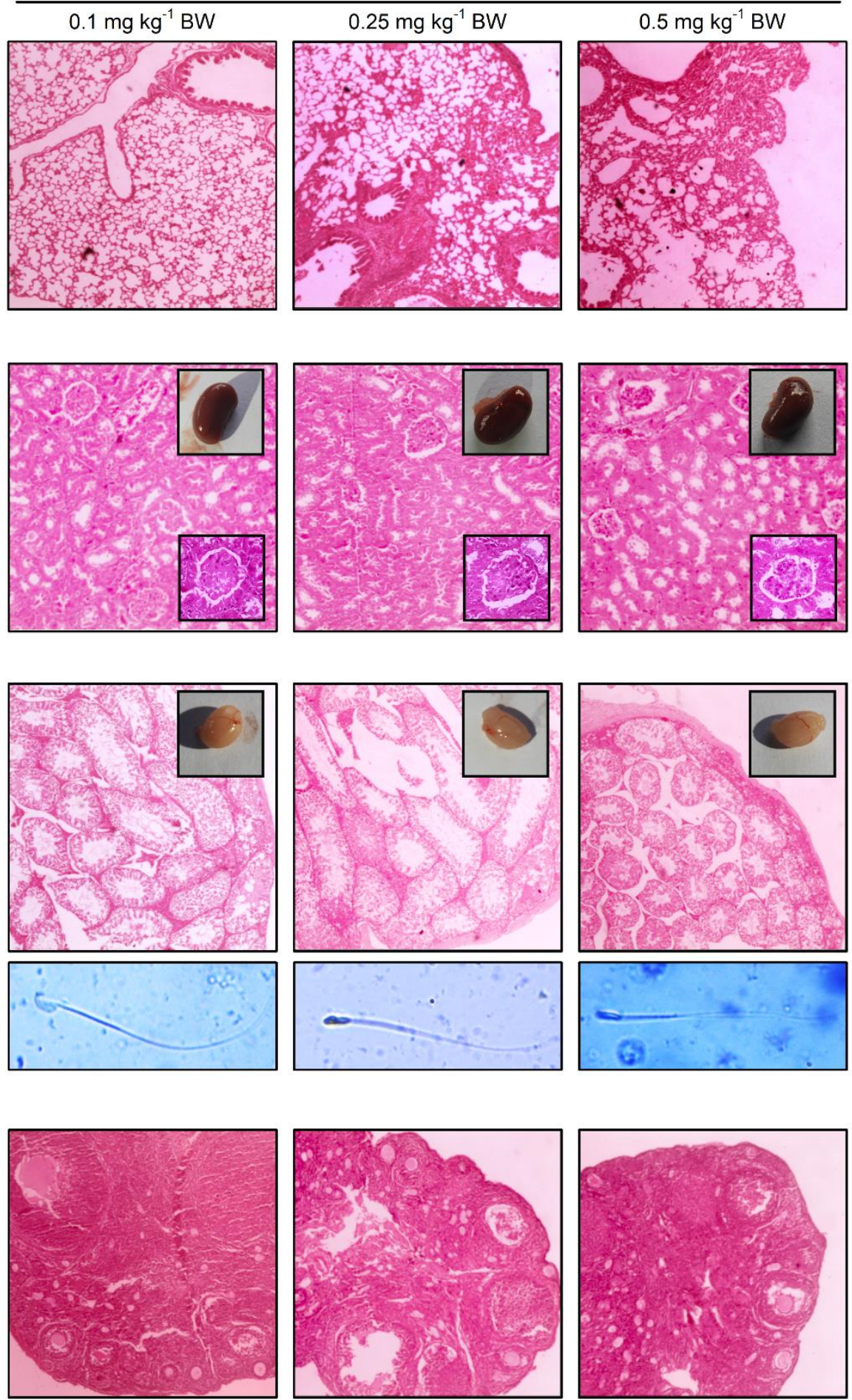

Figure 5. Hematoxylin and eosin stained tissue sections from different groups. (a) Lungs. (b) Kidney. Inset shows morphology of kidney and Bowman's capsule. (c) Testes. Inset shows morphology of testes. Lower pannel shows morphology of sperm from respective groups. (d) Ovary. 